\title{
Experimental characterization of dispersion properties of leaky modes in planar photonic crystal waveguide
}

\author{
Marko Lončar (1), Dušan Nedeljković (2), Thomas P. Pearsall (2), Jelena Vučković (1),
} Axel Scherer (1), Sergey Kuchinsky (3), and Douglas C. Allan (4)

(1) California Institute of Technology, MS 136-93, Pasadena. CA 91125, e-mail: loncar@caltech.edu

(2) CERF, Corning, SA 77210 Avon, France

(3) Corning Scientific Center, 199034 St. Petersburg, Russia

(4) SP-FR-05, Corning Incorporated, Corning. NY 14831

Abstract: We have experimentally observed the coupling of a Bloch wave in a single-line-defect planar photonic crystal and have mapped the dispersion diagram of the leaky mode component of this wave. The results are in excellent agreement with our three dimensional finite difference time domain calculations.

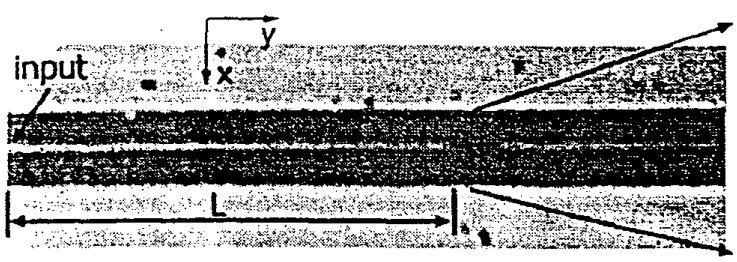

Figure 1. PPC waveguide with discontinuity in the form of single defect cavity.

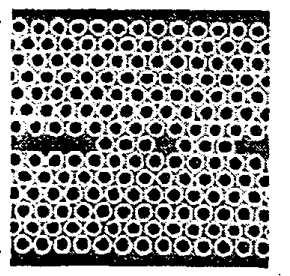

Experimental set-up The planar photonic crystal structure (PPC) that we are interested in is a silicon. slab. suspended in air and perforated with a 2D triangular lattice of holes with radius $r=0.4 \mathrm{a}$. where $\mathrm{a}=530 \mathrm{~nm}$ is the periodicity of the latice. The thickness of the slab is $t=300 \mathrm{~nm}$. The waveguide is defined as a row of missing holes. In this work we have characterized waveguides with discontinuities in the form of a single defect cavity. as shown in Figure 1 . Details on fabrication procedure can be found in our previous publication'. Butt-coupling of a single-mode fiber was used to introduce light from a tunable semiconductor diode laser into the PPC waveguide. Waveguiding performance was observed by visualization of the guiding structure with infrared camera positioned in the plane perpendicular to the sample. This camera was used to detect the light scattered in the vertical direction (out of plane loss). In our previous work ${ }^{2}$ we have used similar setup to analyze waseguides with sharp bends. and we have observed significant out of plane losses when coupling was not optimized. We have attributed those losses to the leak! modes of the waveguide. In this work we investigate this phenomena in more detail.

Experimental observation of radiated leaky modes
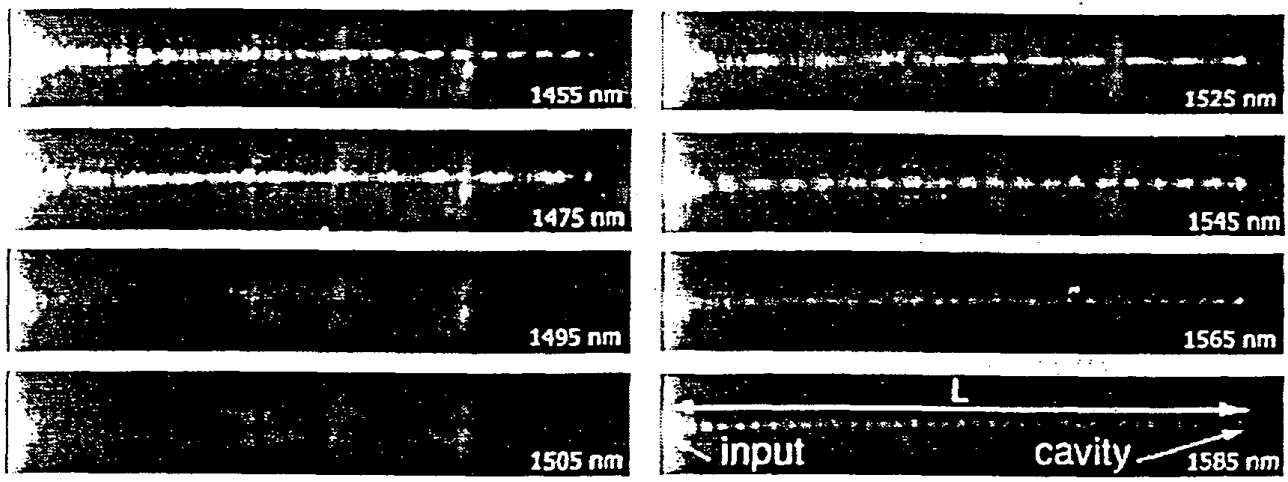

Figure 2. Wavelength dependence of the signal detected by the camera. Periodic modulation pattern can be observed.

In Figure 2 we show signal detected with camera for different wavelengths of the input light. At nearly exery "welength. a clear periodic intensity modulation can be seen along the waveguide direction. Periodicity of this modulation grows shorker is the difference between the wavelength and $1500 \mathrm{~nm}$ grows larger. in either divection of the wavelength. However. for wavelengls in the range $11+95 \mathrm{~nm}$. $150 \mathrm{~nm}$ ) this modulation intensity has nearly disappeared. It is imponant in say that the modulation paltern shown in Figure 2 was detected above the sample surface, that is. at different focal distance irom the 


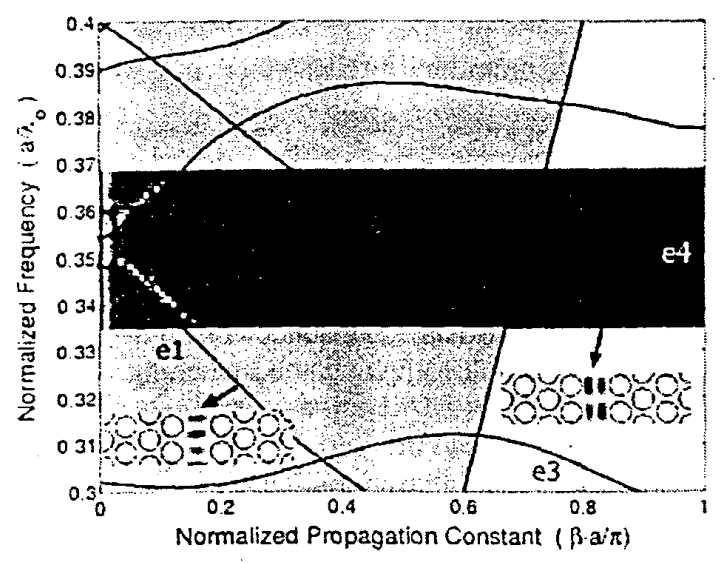

Figure 3. Calculated (black solid lines) and experimentally obtained (white dots in dark gray region) dispersion diagram for the TE-like modes of the PPC waveguide. Mode profiles for some modes are also shown. with PPC at both sides. The launched wave is a Bloch mode consisting of two components. one of which is guided and the other which has a propagation constant close to 0 and is leaky. The standing wave formed by the interference of the leaky mode with itself is responsible for the periodic pattern that we observe. This is very similar to the situation that we have in experiment where waveguide sections are closed with cleaved facet at one side and single defect cavity at the other side. In our experiment cavity acts like mirror since its eigen-mode frequency $(a: \lambda=0.326)$ is outside the frequency range probed in the experiment (dark gray region in Figure 2) and therefore light in the waveguide cannot couple to the caviti mode. Due to the memory limitations of the computers used in the simulations. modeled waveguide sections were about 6 times smaller than the actual structures tested in the experiments. The waveguide sections were excited with dipole source of fixed frequency.

In Figure 4 we show the $P_{z}$ components of the Pointing vector at about lum above the sample surface. for three different normalized frequencies. Clear periodic modulation pattern. very similar to the one observed in the experiment (Figure 2) can be seen. The periodicity of the modulation pattern shows the same trend with respect to the frequencs of light as in the experiment - ior frequencies below the mini stop band. the spatial periodicity grows larger as the frequency increases. Moreover. the spatial profile (not shown here) of the mode excited in the waveguide has the same symmetry as the leaky el mode (Figure 3). thus confirming our hy pothesis of coupling the light into the leaky modes of the structure.

In conclusion. We have experimentalls obtained the dispersion diagram of the leaky modes in the planar photonic crystal waveguide for the wavelengths from $1440 \mathrm{~nm}$ to $1590 \mathrm{~nm}$. A small stop band. around $i=1500 \mathrm{~nm}$ is also detected. The experimentally: obtained results are in ver good agreement with our 3D FDTD calculations.

\section{References}

[1] M. Loncar. T. Doll. J. V'uikovic. A. Scherer. J. of Lightwave Tech.. 18. 1402(2000)

[2] M. Loncar. D. Nedeljkoric. T. Doll. J. Vucković. A. Scherer. T. P. Pearsall. .ippl. Phys. Lett. 77. 1937 (2000) 